\title{
The interacting system AM 1003-435
}

\author{
Guillermo Günthardt ${ }^{1,2}$, Estela L. Agüero ${ }^{1}$, Irapuan Rodrigues ${ }^{3}$ \\ and Rubén J. Díaz ${ }^{1}$ \\ ${ }^{1}$ Observatorio Astronómico de Córdoba, UNC, Laprida 854, 5000 Córdoba, Argentina \\ email: gunth@mail.oac.uncor.edu \\ ${ }^{2} \mathrm{SeCyT}$, Universidad Nacional de Córdoba, Argentina \\ ${ }^{3}$ Instituto de Fisica - UFRGS, CEP 91501-970, Porto Alegre, RS, Brazil
}

\begin{abstract}
We present spectroscopic observations of the peculiar system AM1003-435, which is composed of two strong interacting galaxies, and numerical simulations of the encounter between the galaxies, following the dynamical evolution of the stellar and gaseous components.
\end{abstract}

The most remarkable morphological features of the peculiar system AM 1003-435 are two components or nuclei separated by about $38 "\left(\sim 7.8 \mathrm{kpc}\right.$, considering $75 \mathrm{~km} \mathrm{~s}^{-1}$ $\mathrm{Mpc}^{-1}$ ), connected by an apparent bridge that contains some bright condensations, all this within a diffuse envelope that extends in two long opposed tails. AM 1003-435 was observed in CASLEO, San Juan, Argentina, with a REOSC spectrograph coupled to the $2.15 \mathrm{~m}$ telescope, on April 2, 2003. Spectra were taken with a 2.8 " x 348 " slit in the position angle that connects both nuclei, with a $300 \mathrm{l} \mathrm{mm}^{-1}$ grating covering the wavelength range 4000 - $7500 \AA$. The spectrophotometric characteristics of both nuclei, their neighboring zones and three emission regions in the bridge, are typical of H II regions. Electron temperatures and densities, although low, are in the range of normal values for these kind of regions. The spectra of the NW nucleus present strong emission lines, being the central one typical of a starburst and a continuum dominated by A stars. The spectra of the SE nucleus shows weak emission lines and a continuum corresponding to an advanced stellar spectral type. The three regions associated with the bridge also present emission lines and weak continua. The distribution of oxygen and nitrogen abundances present their highest values in the NW nucleus and decrease with the distance to it, as it was observed in many spiral galaxy discs. In the SE component, the values of the oxygen abundances are comparatively low. In the bridge regions both abundances continue the decreasing tendency already mentioned. For the NW nucleus, the equivalent whidts $\mathrm{EW}(\mathrm{H} \alpha)=119 \AA$ and $\mathrm{EW}(\mathrm{H} \alpha+[N I I])=173 \AA$, are indicative of very intense star formation activity, confirming their starburst nature (Liu \& Kennicutt 1995). For the SE nucleus an $\mathrm{EW}(\mathrm{H} \alpha)=2 \AA$ was found. From these nuclear $\mathrm{H} \alpha$ equivalent widths we derived indicative ages for their respective star formation bursts: $6.5 \times 10^{6}$ years for the NW nucleus and $2.1 \times 10^{7}$ years for the SE one, as resulted from the standard model for instantaneous bursts with metallicity of $0.6 \mathrm{Z}_{\odot}$ and $0.3 \mathrm{Z}_{\odot}$ (Leitherer et al. 1999). The resulting abundances and ages suggest that the starburst in the NW nucleus was triggered in a richer medium than in the SE nucleus. The integrated spectrum of the NW component, is typical of a starburst (Liu \& Kennicutt 1995), with $\mathrm{EW}(\mathrm{H} \alpha)=$ $87 \AA$ and $\mathrm{EW}(\mathrm{H} \alpha+[N I I])=126 \AA$, corresponding to a Sc/Sm galaxy. The integrated spectrum of the SE component, characteristic of slightly star forming systems (Liu \& Kennicutt 1995), corresponds to Sa-Sab type galaxies. From the integrated spectrum of $\mathrm{AM} 1003-435$ we derived, $\mathrm{EW}(\mathrm{H} \alpha)=46 \AA$ and $\mathrm{EW}(\mathrm{H} \alpha+[N I I])=62 \AA$, which reflects the merger nature of this system (Liu \& Kennicutt 1995), like its morphology suggests. 


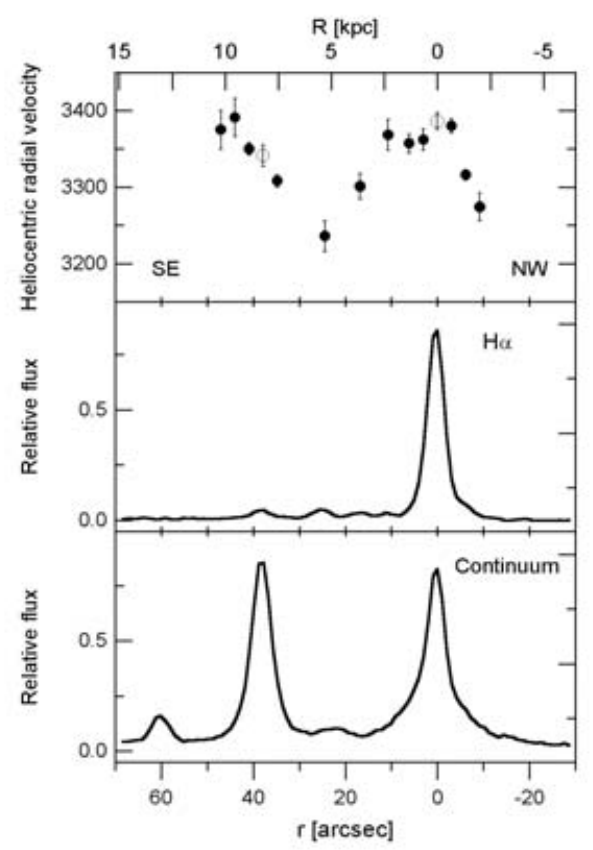

Figure 1. Upper panel: Radial velocity distribution across the position angle that connects the nuclei (open circles correspond to both nuclei). Middle panel: $\mathrm{H} \alpha$ flux distribution (continuum subtracted). Lower panel: Continuum distribution.

The velocity distribution (fig. 1) presents strong distortions with an important gradient of $44 \mathrm{~km} \mathrm{~s}^{-1} \mathrm{kpc}^{-1}$ along the tidal bridge. In fig. 1, the $\mathrm{H} \alpha$ flux distribution is also shown (continuum subtracted) and the continuum distribution (the signal at 60" correspond to a star), where it is observed, besides of the localized stellar contribution, that the NW component presents a well traced bulge and disk, while that in the SE we observed only an important bulge, indicative of an earlier morphological type, what reinforces the classification made before. In order to understand the dynamical evolution of AM 1003435, the encounter was simulated the encounter, following the evolution of the stellar and gaseous content of the galaxies. A complete set of low resolution simulations were done using Hernquist \& Katz (1989) TreeSPH code. Galaxy models were constructed following the general prescription of Hernquist (1993) including a gas disk component. The simulations suggest that the perigalacticum occurred 150 Myr ago. Star formation in both galaxies would have begun after the perigalacticum. The eastern tail would belong to the SE progenitor and would be bluer than the western one, due to the light content of disk stars of the less massive and shattered SE body. In this kind of scenario, the galaxies will finally merge (nucleus-nucleus distance less than $1 \mathrm{kpc}$ ) in about $8 \times 10^{8} \mathrm{yr}$.

In this system, the interaction does not seem to be feeding a black hole neither nuclear activity.

\section{References}

Liu, Ch. T., \& Kennicutt, R. C. 1995, ApJ, 450, 547

Hernquist, L., \& Katz, N. 1989, ApJS, 70, 419

Hernquist, L. 1983, ApJS, 86, 389

Leitherer, C., et al. 1999, ApJS, 79, 255 